\title{
Carbonyl Cyanide 3-Chlorophenylhydrazone (CCCP) Exhibits Direct Antibacterial Activity Against Mycobacterium abscessus
}

This article was published in the following Dove Press journal: Infection and Drug Resistance

\author{
Suting Chen ${ }^{1, *}$ \\ Tianlu Teng ${ }^{1,2}, *$ \\ Zhuman Zhang' \\ Yuanyuan Shang ${ }^{1,2}$ \\ Hua Xiao' \\ Guanglu Jiang' \\ Fen Wang' \\ Junnan Jia \\ Lingling Dong' \\ Liping Zhao' \\ Naihui Chu ${ }^{2}$ \\ Hairong Huang'
}

'National Clinical Laboratory on Tuberculosis, Beijing Key Laboratory for Drug Resistant Tuberculosis Research, Beijing Chest Hospital, Capital Medical University, Beijing Tuberculosis and

Thoracic Tumor Institute, Beijing, People's Republic of China; ${ }^{2}$ Department of Tuberculosis; Beijing Chest Hospital, Capital Medical University, Beijing Tuberculosis and Thoracic Tumor Institute, Beijing, People's Republic of China

*These authors contributed equally to this work
Correspondence: Hairong Huang; Naihui Chu

Tel +86-10-89509|59; +86-10-8950930I

Email nclhuang@ccmu.edu.cn;

dongchu1994@sina.com
Objective: Treatment choices for Mycobacterium abscessus (M. abscessus) infections are very limited, and the prognosis is generally poor. Effective new antibiotics or repurposing existing antibiotics against $M$. abscessus infection are urgently needed. Carbonyl cyanide 3-chlorophenylhydrazone (CCCP), a member of the lipophilic weak acid class, is known as an efflux pump inhibitor for Mycobacterium tuberculosis. The aim of this study was to determine the inhibitory activity of CCCP as a potential novel antibiotic against M. abscessus.

Methods: A total of 47 reference strains of different mycobacterial species and 60 clinical isolates of M. abscessus were enrolled. In vitro inhibitory activity of CCCP was accessed using microplates alamar blue method with the reference and clinical isolates. The activity of CCCP against intracellular M. abscessus residing within macrophage was also evaluated by intracellular colony numerating assay.

Results: CCCP exhibited good activity against $M$. abscessus clinical isolates in vitro, the minimum inhibitory concentration (MIC) ranged from $0.47 \mu \mathrm{g} / \mathrm{mL}$ to $3.75 \mu \mathrm{g} / \mathrm{mL}$, with a $\mathrm{MIC}_{50}$ of $1.875 \mu \mathrm{g} / \mathrm{mL}$ and $\mathrm{MIC}_{90}$ of $3.75 \mu \mathrm{g} / \mathrm{mL}$. At concentrations safe for the cells, CCCP exhibited highly intracellular bactericidal activities against $M$. abscessus and $M$. massiliense reference strains, with inhibitory rates of $84.8 \% \pm 8.8 \%$ and $72.5 \% \pm 13.7 \%$, respectively. CCCP demonstrated bactericidal activity against intracellular $M$. abscessus that was comparable to clarithromycin, and concentration-dependent antimicrobial activity against $M$. abscessus in macrophages was observed. In addition, CCCP also exhibited good activities against most reference strains of rapidly growing mycobacterial species.

Conclusion: CCCP could be a potential candidate of novel antimicrobiological agent to treat M. abscessus infection.

Keywords: Mycobacterium abscessus, carbonyl cyanide 3-chlorophenylhydrazone, minimum inhibitory concentration, intracellular bactericidal activity

\section{Introduction}

Non-tuberculous mycobacteria (NTM) are opportunistic pathogens that exist ubiquitously in soil and water sources and usually cause serious lung infections in vulnerable population. ${ }^{1}$ Human lung infections caused by NTM have rapidly increased globally in the past decades. ${ }^{2,3}$ Among NTM species, Mycobacterium abscessus (M. abscessus) is one of the most frequently identified species with clinical significance. ${ }^{4} M$. abscessus belongs to the fast-growing group of NTM that cause skin and soft tissue infections beside lung infection. It is estimated that 
this species caused 5-20\% of all non-Mycobacterium tuberculosis (Mtb) infections globally. Furthermore, this rate is likely to be an underestimation since most of the countries do not routinely report these infectious diseases. The treatment of $M$. abscessus caused infections requires combinational usage of some of the following antibiotics: macrolide antibiotics, amikacin, cefoxitin and other drugs. However, poor prognosis of treatment is frequently confronted, whereas the cure rate only reaches $25-58 \%{ }^{5,6}$ As a consequence, $M$. abscessus infection is often considered as "antibiotic nightmare". 7 Therefore, new or repurposed drugs are urgently needed to change this clinical predicament.

Carbonyl cyanide 3-chlorophenylhydrazone (CCCP) functions as a proton carrier that reduces the transmembrane electrochemical gradient and causes an accumulation of antibiotics within bacterial cells. However, CCCP has generally been used only as an efflux pump inhibitors (EPIs). ${ }^{8-10} \mathrm{CCCP}$ has been proven to reduce the minimum inhibitory concentration (MIC) of rifampicin, isoniazid for $\mathrm{Mtb}^{11}$ and improved the activity of clarithromycin (CLA) against CLA-resistant $M$. abscessus clinical strains. ${ }^{12}$ It is generally deem that CCCP, as an EPIs, is more effective when used in combination with antibiotics. ${ }^{13}$ We identified that CCCP alone had antibacterial activity against M. abscessus and possessed the potential to be considered as an independent antibiotic. In this study, we determined the susceptibility profile of $M$. abscessus clinical isolates to CCCP, investigated its bactericidal activity against M. abscessus in macrophages, and also analyzed its cytotoxicity. To our knowledge, this is the first report on the independent anti-M. abscessus activity of CCCP as a novel candidate antibiotic.

\section{Materials and Methods}

\section{Ethical Approval}

The study was approved by the Ethics Committee of Beijing Chest Hospital, Capital Medical University. All the reference strains and clinical strains were collected from the Beijing biobank of Clinical Resources on Tuberculosis located in Beijing Chest Hospital, Capital Medical University (Beijing, China); therefore the informed consents were waived.

\section{Bacterial Strains and Culture}

The M. abscessus clinical isolates were originally isolated from sputa of patients with lung infections. The strains were classified as NTM preliminarily with p-nitrobenzoic acid containing medium, and later identified into species by sequence alignments of $16 S \mathrm{rRNA}$, hsp65, rpoB, 16$23 S$ rRNA internal transcribed spacer in parallel. The reference strains including 29 rapidly growing mycobacteria (RGM) species, 18 slowly growing mycobacteria (SGM) species were obtained either from the American Type Culture Collection (ATCC) or from the German Collection of Microorganisms (DSM). All the bacterial strains studied were stored at $-80^{\circ} \mathrm{C}$ in the Bio-bank in Beijing Chest Hospital, including reference strains and a total of $60 \mathrm{M}$. abscessus clinical isolates. M. abscessus clinical isolates consist of 40 M. abscessus subsp. abscessus and $20 \mathrm{M}$. abscessus subsp. massiliense. Bacterial suspensions were inoculated on the Middlebrook $7 \mathrm{H} 10$ (M7H10) agar plates or on the Lowenstein-Jensen (LJ) medium. The growth phenotypes of the clinical strains on the L-J medium were recorded.

\section{Antimicrobial Susceptibility Testing}

CCCP (CAS\#555-60-2) and CLA (CAS\#81103-11-9) were purchased from Sigma-Aldrich Co. (St Louis, MO, USA) or Solarbio (Beijing, China), respectively. Both drugs were dissolved in dimethyl sulfoxide (DMSO) at a concentration of $2400 \mu \mathrm{g} / \mathrm{mL}$. MICs were determined according to the guidelines from the Clinical and Laboratory Standards Institute (CLSI). ${ }^{14}$ Cation-adjusted Mueller-Hinton broth (CAMHB) (BD, USA) enriched with 5\% OADC was used for SGM, while CAMHB without OADC was used for RGM. CCCP was serially diluted (1:2) with final concentrations ranging from 0.115 to 60 $\mu \mathrm{g} / \mathrm{mL}$. NTM cultures were scraped from LJ media, homogenized and then adjusted to McFarland 0.5 with sterile saline, and then diluted 200-fold for inoculation. One hundred $\mu \mathrm{L}$ of diluted inoculum was added into each well. The plates were incubated at $37{ }^{\circ} \mathrm{C}$ for 3 to 4 days for RGM and 7 to 8 days for SGM until visible growth of mycobacteria in the control wells without CCCP. Twenty $\mu \mathrm{L}$ of alamarBlue (Invitrogen) and $50 \mu \mathrm{L}$ of $5 \%$ Tween 80 were added to each well and the plates were re-incubated at $37^{\circ} \mathrm{C}$ for additional $24 \mathrm{~h}$ before assessing color change. Blue color indicated no mycobacterial growth and pink color indicated mycobacterial growth. The MIC was defined as the lowest drug concentration that inhibited the bacterial growth, observed by no color change from blue to pink. The MIC testing for each strain was performed in triplicate. 


\section{Minimum Bactericidal Concentration (MBC) Against M. abscessus}

The reference strains and three clinical isolates for each of M. abscessus subsp. abscessus or subsp. massiliense were randomly selected for the assay. After 4 days' incubation with CCCP at different concentrations, the wells in which the drug concentrations were higher than the MIC were resuspended and $100 \mu \mathrm{L}$ medium of each well was inoculated onto 7H10-OADC plate. The plates were incubated at $37^{\circ} \mathrm{C}$ for 5 days, followed by counting the number of colonies. The MBC value was defined as the minimum drug concentration with no colony growth. An antibiotic was considered bactericidal when the $\mathrm{MBC} / \mathrm{MIC}$ ratio was $\leq 4$; otherwise, it was considered bacteriostatic. ${ }^{15}$

\section{Cytotoxicity Assay}

The toxicity of CCCP was assessed using THP-1 and U937 cells which were purchased from the Wuhan Procell Life Science\&Technology Co., Ltd. Cells were seeded into 24-well plates and differentiated into macrophages with $100 \mathrm{nM}$ PMA. After $24 \mathrm{~h}$, cells were washed once and cultured in fresh RPMI medium (Gibco) with $10 \%$ fetal bovine serum (RPMI complete medium). CCCP solution was added into the wells with final concentrations ranging from 0.78 to $25 \mu \mathrm{g} / \mathrm{mL}$, and incubated for additional $48 \mathrm{~h}$. The same volume of DMSO was used to replace $\mathrm{CCCP}$ as control. The cytotoxicity of different concentrations of CCCP was monitored using a lactate dehydrogenase (LDH) cytotoxicity detection kit (Beyotime, Shanghai, China). The LDH activity in the cell culture supernatant were analysed and the absorbance value (Abs) under 490nm was recorded using Multiskan Go microplate reader (Thermo Fisher, USA). The cell survival rate (\%), at each concentration was determined as follows: cell survival rate $=(1-($ Abs 490 of treated cellsAbs490 of control cells)/(Abs490 of total lysis cellsAbs490 of control cells) $) \times 100 \%$.

\section{Intracellular Killing and Concentration-Kill Assay}

U937 cells were seeded at $2 \times 10^{5}$ cells/well in a 24 -well plate and differentiated with PMA. Cells were infected with M. abscessus reference strain (ATCC 19977) and M. massiliense reference strain (CCUG 48898) at a multiplicity of infection (MOI) of 1 or 10 . At $2 \mathrm{~h}$ postinfection, the cells were gently washed three times with pre-warmed phosphate-buffered saline (PBS) to remove the extracellular bacteria. RPMI complete medium containing $250 \mu \mathrm{g} / \mathrm{mL}$ amikacin were added to kill the remaining extracellular bacteria for an additional $2 \mathrm{~h}^{16}$ For intracellular killing assay, RPMI complete medium containing CCCP $(6 \mu \mathrm{g} / \mathrm{mL})$ or CLA $(2 \mu \mathrm{g} / \mathrm{mL})$ were added. Meanwhile, RPMI complete medium containing different concentrations of CCCP were supplied for the concentration killing assay. The culture medium with vehicle (DMSO) was included as growth controls. At $48 \mathrm{~h}$ postinfection, macrophages were extensively washed with PBS and lysed with $0.1 \%$ Tween 80 , and serially diluted with $0.05 \%$ Tween 80 . The number of CFUs was quantified by plating serial dilutions of lysates on M7H10 agar plates. The bacterial survival rate was calculated using the formula: viability $=\mathrm{CFU}$ of bacteria under the treatment of CCCP or CLA/CFU of bacteria treated with vehicle $\mathrm{DMSO} \times 100 \%$.

\section{Statistical Analysis}

Data were analyzed using SPSS 24.0 software and GraphPad Prism 8.0 software. For antimicrobial susceptibility assay, non-parametric test was used to determine significant differences between groups. For cytotoxicity assay and intracellular bactericidal assay, one-way analysis of variance (ANOVA), followed by post hoc test, was used to determine significant differences between the groups. Differences were considered to be statistically significant for $p$ value of $<0.05$.

\section{Results}

\section{Activity Spectra of CCCP Against NTM Reference Strains}

The MICs of CCCP against the 47 reference strains are shown in Table 1. CCCP showed excellent activities against most of the RGM reference strains. However, four RGM species had MICs of $3.75 \mu \mathrm{g} / \mathrm{mL}$ and $7.5 \mu \mathrm{g} /$ $\mathrm{mL}$, and 24 other RGM species had MICs of $\leq 1.875 \mu \mathrm{g} /$ $\mathrm{mL}$. Additionally, all of the 18 enrolled SGM species had MICs of $\geq 7.5 \mu \mathrm{g} / \mathrm{mL}$.

\section{MICs and MBCs of CCCP Against}

\section{M. abscessus Isolates}

A total of $60 \mathrm{M}$. abscessus clinical isolates including 40 M. abscessus subsp. abscessus and 20 M. abscessus subsp. massiliense, were studied. The morphological observation indicated that 22 out of the 40 isolates of M. abscessus subsp. abscessus had smooth morphotypes, while 18 
Table I MICs of CCCP for Reference Strains of 29 RGM Species and 18 SGM Species

\begin{tabular}{|c|c|c|}
\hline Strain by Type & $\begin{array}{l}\text { Mycobacterium Species } \\
\text { (Strain) }\end{array}$ & MIC $(\mu g / m L)$ \\
\hline \multicolumn{3}{|l|}{ RGM species } \\
\hline ATCC 19977 & M. abscessus & 0.94 \\
\hline CCUG 48898 & M. massilliense & 0.94 \\
\hline ATCC 33464 & M. austroafricanum & 0.94 \\
\hline ATCC 70073I & M. septicum & 1.875 \\
\hline ATCC 35796 & M. senegalense & 0.94 \\
\hline ATCC 27406 & M. agri & 0.47 \\
\hline ATCC 19527 & M. thermoresistibile & 0.94 \\
\hline ATCC 27278 & M. chubuense & 0.94 \\
\hline ATCC 35154 & M. pulveris & 0.94 \\
\hline ATCC 19627 & M. chitae & 0.94 \\
\hline ATCC 4327I & M. peregrinum & 1.875 \\
\hline ATCC 19686 & M. parafortuitum & 0.47 \\
\hline ATCC I5483 & M. vaccae & 7.5 \\
\hline ATCC II 758 & M. phlei & 0.47 \\
\hline ATCC I 4474 & M. flavescens & 0.47 \\
\hline ATCC 19340 & M. diernhoferi & 0.94 \\
\hline ATCCI4472 & M. chelonae & 0.94 \\
\hline ATCC 684I & M. fortuitum & 3.75 \\
\hline ATCC 25795 & M. neoaurum & 1.875 \\
\hline ATCC 33776 & M. porcinum & 1.875 \\
\hline DMS 44I 24 & M. mucogenicum & 1.875 \\
\hline ATCC BAA-955 & M. goodii & 1.875 \\
\hline ATCC 43909 & M. gilvum & 0.23 \\
\hline ATCC 44829 & M. cosmeticum & 3.75 \\
\hline ATCC 19420 & M. smegmatis & 7.5 \\
\hline ATCC 23366 & M. aurum & 0.94 \\
\hline ATCC 27280 & M. aichiense & 0.94 \\
\hline ATCC 27023 & M. obuense & 0.94 \\
\hline ATCC 35219 & M. fallax & 1.875 \\
\hline \multicolumn{3}{|l|}{ SGM species } \\
\hline ATCC 27294 & Mtb H37Rv & 15 \\
\hline ATCC 19422 & M. microti & 15 \\
\hline ATCC 15754 & M. gastri & 15 \\
\hline ATCCI3950 & M. intracellulare & 7.5 \\
\hline ATCC 14470 & M. gordonae & 7.5 \\
\hline ATCC 12478 & M. kansasii & 7.5 \\
\hline ATCC 35799 & M. szulgai & 15 \\
\hline ATCC 2529I & M. avium & 15 \\
\hline DSM 44648 & M. parascrofulaceum & 15 \\
\hline ATCC 51131 & M. celatum & 15 \\
\hline ATCC 1998I & M. scrofulaceum & 7.5 \\
\hline ATCC 27726 & M. gadium & 7.5 \\
\hline ATCC 19250 & M. xenopi & 15 \\
\hline ATCC 27024 & M. rhodesiae & 30 \\
\hline ATCC 19530 & M. nonchromogenicum & 60 \\
\hline ATCC 25276 & M. asiaticum & 15 \\
\hline ATCC 15755 & M. terrae & 15 \\
\hline ATCC 23292 & M. triviale & 7.5 \\
\hline
\end{tabular}

exhibited rough morphotypes. Similarly, 11 of the 20 M. abscessus subsp. massiliense isolates exhibited smooth colonies and 9 exhibited rough colonies. The MICs of CCCP against $M$. abscessus (ATCC19977) and M. massiliense (CCUG48898) were both $0.94 \mu \mathrm{g} / \mathrm{mL}$. The MICs of CCCP against M. abscessus clinical isolates ranged from 0.47 to $3.75 \mu \mathrm{g} / \mathrm{mL}$, the majority of these ranged from 0.9375 to $1.875 \mu \mathrm{g} / \mathrm{mL}$ (Figure 1A). The $\mathrm{MIC}_{50}$ and $\mathrm{MIC}_{90}$ of CCCP against M. abscessus subsp. abscessus were both $1.875 \mu \mathrm{g} / \mathrm{mL}$. The $\mathrm{MIC}_{50}$ and $\mathrm{MIC}_{90}$ of CCCP against $M$. abscessus subsp. massiliense were 1.875 and $3.75 \mu \mathrm{g} / \mathrm{mL}$, respectively. In contrast with M. abscessus subsp. abscessus, M. abscessus subsp. massiliense showed greater MIC to CCCP $(P<0.05)$ (Figure 1C). Additionally, the MICs of CCCP against $M$. abscessus rough morphotype were greater than these of $M$. abscessus smooth morphotype $(P<0.05)$ (Figure 1D). Specifically, the M. abscessus subsp. abscessus with smooth morphotype was the most sensitive group whereas the $M$. abscessus subsp. massiliense with rough morphotype was the most tolerant group to CCCP exposure (Figure 1B). The $\mathrm{MBC} / \mathrm{MIC}$ ratio of $\mathrm{CCCP}$ against M. abscessus reference strains and 6 clinical isolates ranged from 4 to 8 (Table 2). Furthermore, 4 strains had $\mathrm{MBC} / \mathrm{MIC}$ ratios $>4$, one strain belonged to M. abscessus subsp. abscessus with smooth morphotype and another one belonged to $M$. abscessus subsp. massiliense with rough morphotype had $\mathrm{MBC} / \mathrm{MIC}$ ratios $=4$.

\section{Cytotoxicity Assay of CCCP in the THP-I and U937 Cells}

When the concentration of CCCP was lower than $6.25 \mu \mathrm{g} /$ $\mathrm{mL}$, the survival rates for both THP-1 and U937 cells were almost 97\%. However, with the concentration of CCCP at $12.5 \mu \mathrm{g} / \mathrm{mL}$ or greater, the cells survival rates were less than $80 \%$ (Figure 2).

\section{Intracellular Bactericidal Activity of CCCP Against M. abscessus in Macrophages}

Bacterial survival rate was quantitated as shown in Figure 3. Both at $\mathrm{MOI}=1: 1$ and $\mathrm{MOI}=1: 10$, obvious reductions were observed in the colony number of $M$. abscessus $(\mathrm{CFU} / \mathrm{mL})$ treated with CCCP group $(6 \mu \mathrm{g} / \mathrm{mL})$ compared to the DMSO group. The intracellular growth inhibition rates of CCCP against both M. abscessus and 

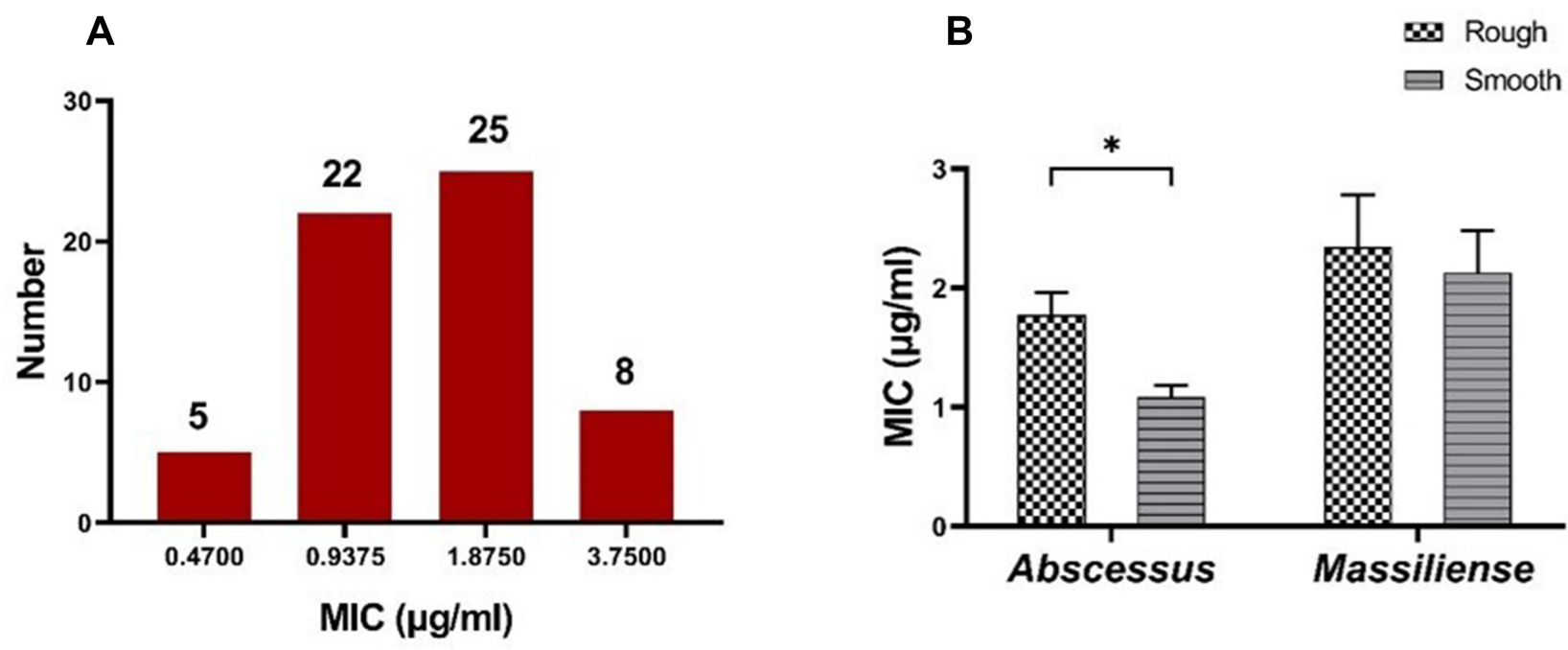

C
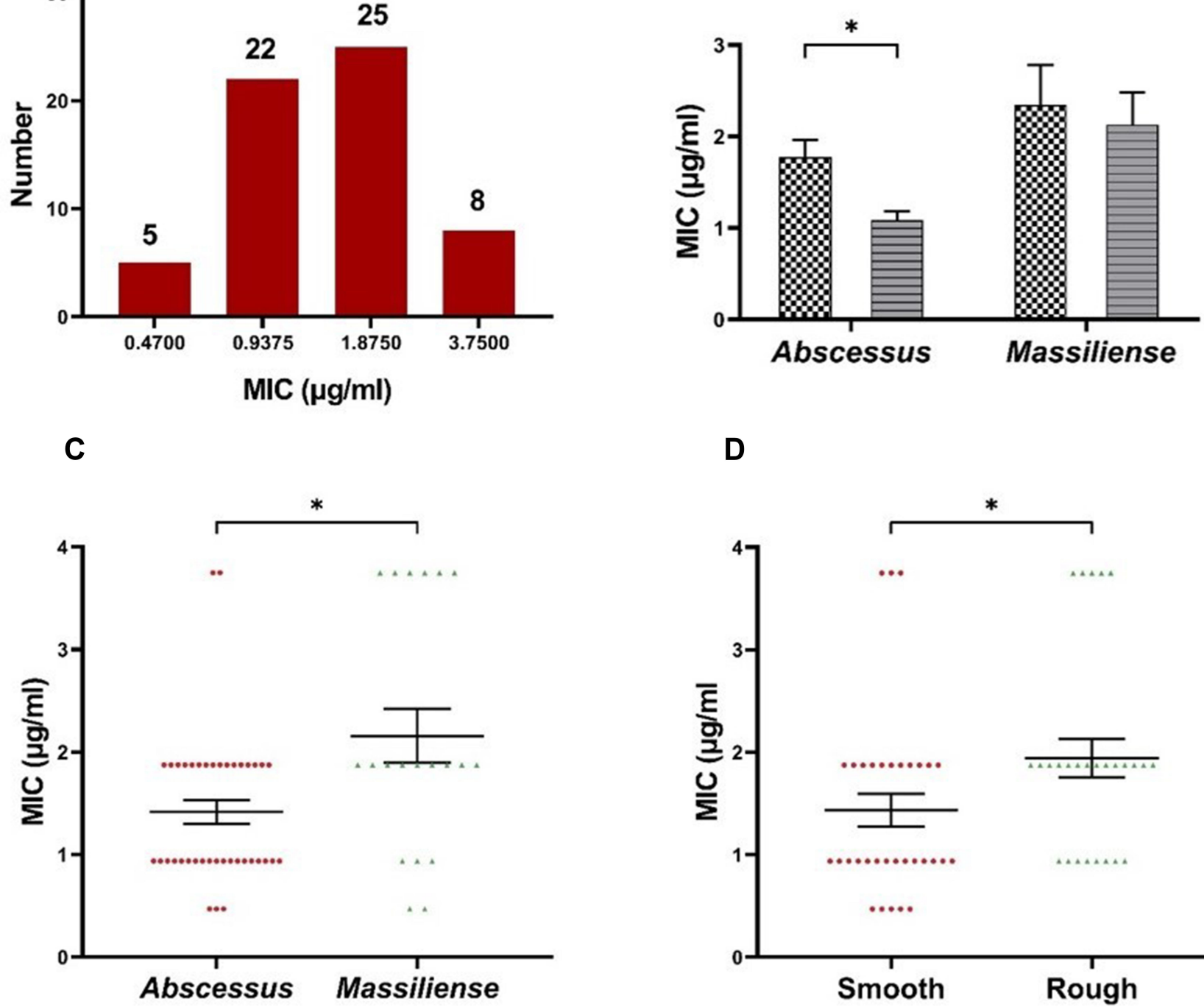

D

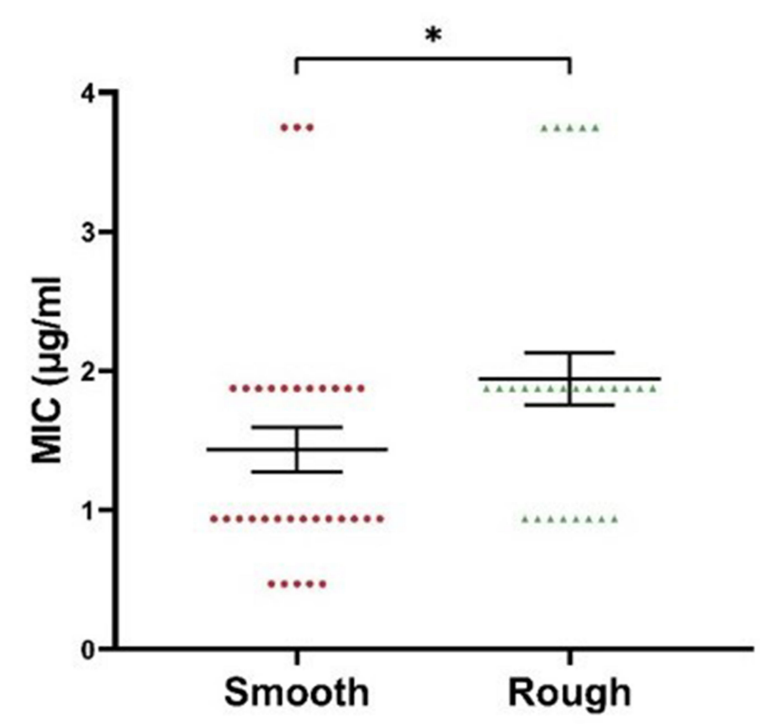

Figure I The MIC of CCCP against 60 clinical M. abscessus isolates. (A) The MIC distribution of the M. abscessus isolates. (B) The MICs of CCCP in rough morphotype and smooth morphotype. (C) The MIC distribution of the isolates of M. abscessus subspecies abscessus and M. abscessus subspecies massiliense. (D) The MIC distribution of the isolates with smooth morphotype or rough morphotype. Error bars represent the standard errors of the data points. $* P<0.05$.

M. massiliense reference strains at MOI of 10 were significantly higher than MOI of 1 . Specifically, at MOI of 10, CCCP inhibited $84.8 \% \pm 8.8 \%$ of intracellular bacterial growth against $M$. abscessus and $72.5 \% \pm 13.7 \%$ of intracellular bacterial growth against M. massiliense. Similarly, compared with DMSO group, a pronounced decrease was observed in the colony number of $M$. abscessus treated with CLA group ( $2 \mu \mathrm{g} / \mathrm{mL}$ ) (with inhibitory rates of $92.0 \%$ $\pm 4.0 \%$ in $M$. abscessus and $76.4 \% \pm 11.7 \%$ in M. massiliense, respectively) and CCCP + CLA group (3 $\mu \mathrm{g} / \mathrm{mL}$ CCCP and $1 \mu \mathrm{g} / \mathrm{mL}$ CLA) (with inhibitory rates of $90.4 \% \pm 5.6 \%$ in $M$. abscessus and $79.4 \% \pm 4.9 \%$ in M. massiliense, respectively). By gradually increasing the
CCCP concentration, the intracellular bactericidal activity enhanced linearly, and uniform tendencies were observed at the MOI of 1 and 10 (Figure 4).

\section{Discussion}

Due to the intrinsic resistance of $M$. abscessus to most antibiotics, treatment of M. abscessus infections is highly challenging and often results in failure. ${ }^{17}$ Previous studies have shown that CCCP as an EPI can reduce the drug tolerance level of Mtb and NTM. ${ }^{11-13}$ However, antibacterial activity of CCCP as an independent antibiotic has never been reported. In this study, we evaluated the in vitro efficacy of CCCP against 46 NTM reference 
Table 2 MIC, MBC Values and Antibacterial Activities of CCCP Against M. abscessus Complex

\begin{tabular}{|l|l|l|c|c|}
\hline Isolate & Subspecies & Morphotype & MBC/MIC Ratio & Antibacterial Activity \\
\hline ATCCI9977 & abscessus & Smooth & 8 & Bacteriostatic \\
\hline 164 & abscessus & Rough & 8 & Bacteriostatic \\
\hline 170 & abscessus & Rough & 8 & Bacteriostatic \\
\hline 276 & abscessus & Smooth & 8 & Bactericidal \\
\hline CCUG 48898 & massiliense & Smooth & 8 & Bacteriostatic \\
\hline 131 & massiliense & Rough & 4 & Bacteriostatic \\
\hline 122 & massiliense & Rough & 8 & Bacteriostatic \\
\hline 274 & massiliense & Smooth & 8 & 8 \\
\hline
\end{tabular}

strains and observed that CCCP presented good activities against most of the RGM reference strains, including the notorious $M$. abscessus species. Then, we specifically tested more clinical M. abscessus isolates and surprisingly found that CCCP showed excellent intracellular bactericidal activity against $M$. abscessus. Moreover, variable inhibitory activities were presented against different subspecies or different morphotypes of $M$. abscessus. The M. abscessus subsp. abscessus with smooth morphotype was the most sensitive group to $\mathrm{CCCP}$ exposure while the $M$. abscessus subsp. massiliense was the vice versa. The MBC/MIC ratio of CCCP for M. abscessus is mainly indicative of bacteriostatic activity, although bactericidal activity might also be possible for the minority population strains of these species. The most important finding of our study is that CСCP has a strong intracellular bactericidal activity and this activity is concentrationdependent. The above results indicate that CCCP could be a promising drug candidate for the treatment of M. abscessus and worthy of further investigation.

In this study, RGM and SGM showed very different susceptibility profiles to CCCP treatment in vitro. CCCP presented good inhibitory activities against most of the enrolled RGM reference strains, but evidently weaker inhibitory activities against the enrolled SGM reference species. We speculate that the different growth and metabolism characteristics of RGM and SGM might cause this discrepancy. However, the mechanism underlying this phenomenon remains to be elucidated. Among the other enrolled RGM reference strains, $M$. chelonae had MIC to $\mathrm{CCCP}$ at $0.94 \mu \mathrm{g} / \mathrm{mL}$, whereas M. fortuitum, another frequently isolated RGM species with clinical significance,
A

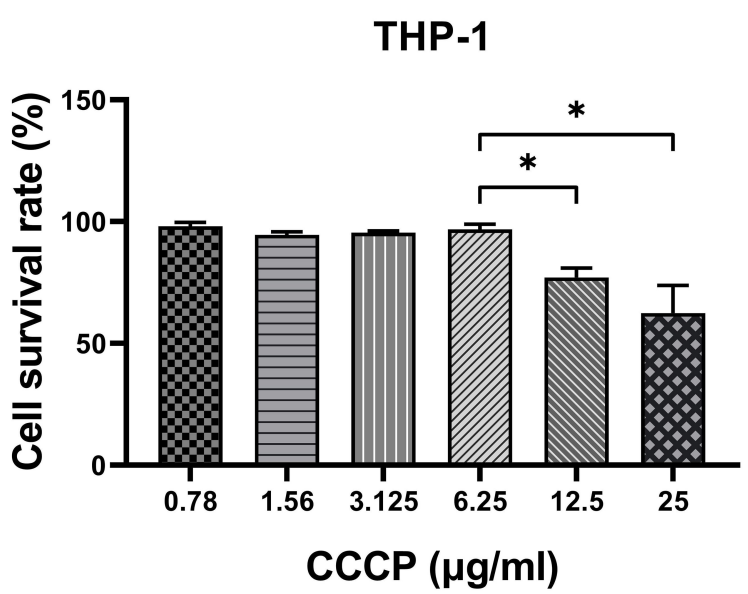

B

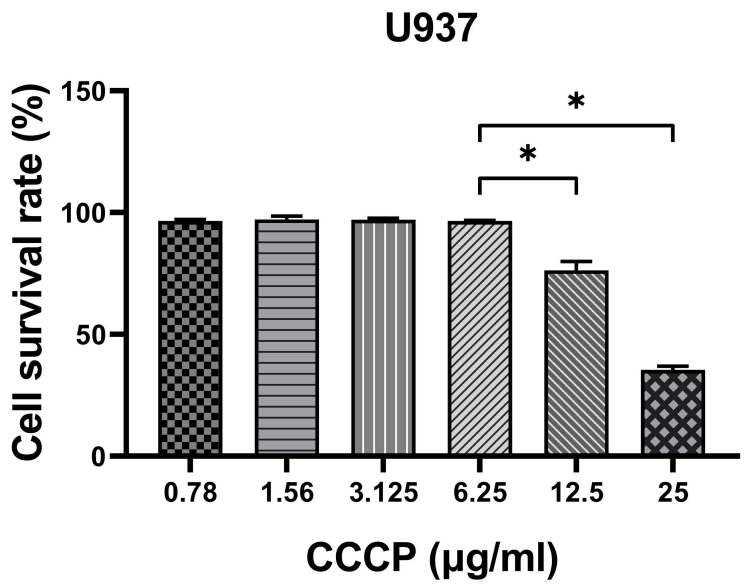

Figure 2 Cytotoxicity assay of CCCP in the THP-I and U937 cells. THP-I cells (A) and U937 cells (B) were treated with different concentrations of CCCP and LDH activity in the cell culture supernatant were analysed. The experiments were carried out in quintuplicate. All data are shown as the means \pm SD ( $n=5)$. Results shown are from one representative experiment, $* \mathrm{P}<0.05$. 
A

\section{MOI 1:1}

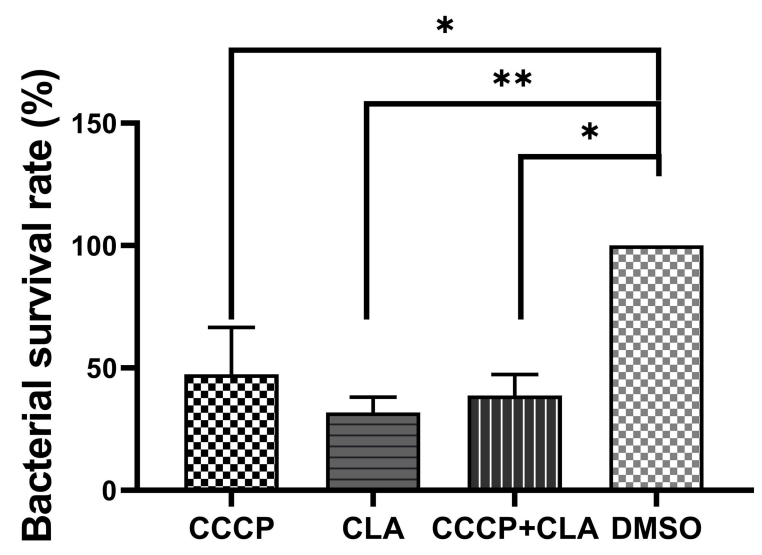

M. abscessus

C

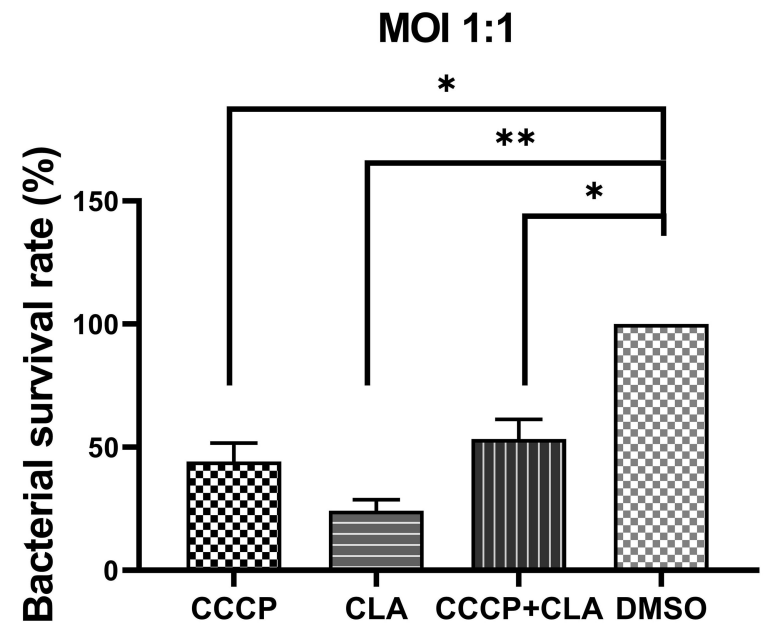

M. massiliense
B

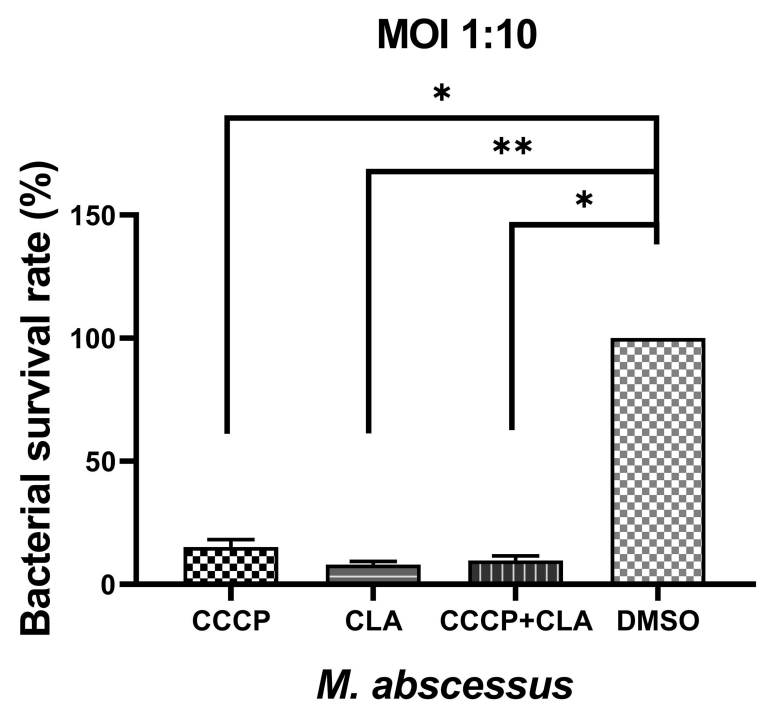

D

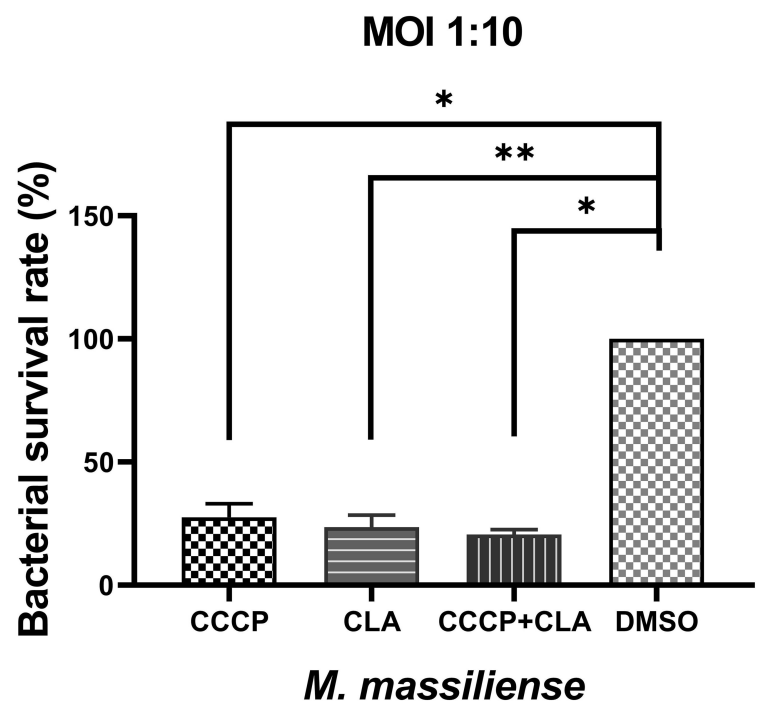

Figure 3 Intracellular bacterial survival rate of M. abscessus (ATCC 19977) (A and B) and M. massiliense (CCUG 48898) (C and D) with CCCP treatment. CCCP group: infected macrophage treated with CCCP $(6 \mu \mathrm{g} / \mathrm{mL})$; CLA group: infected macrophage treated with CLA $(2 \mu \mathrm{g} / \mathrm{mL})$; CCCP+CLA group: infected macrophage treated with $\operatorname{CCCP}(3 \mu \mathrm{g} / \mathrm{mL})$ and CLA $(\mathrm{I} \mu \mathrm{g} / \mathrm{mL})$. The CFU were enumerated in triplicates. All data are shown as the means $\pm S D(n=3)$. $* P<0.05 ; * * P<0.00 \mathrm{I}$.

had MIC at $3.75 \mu \mathrm{g} / \mathrm{mL}$. This diversity within RGM is also worthy of further study.

Our study focused on evaluation of the antimicrobial activity against $M$. abscessus, which is one of the most frequently isolated NTM species with treatment difficulty. CCCP demonstrated good antimicrobial activities against both the reference strains and clinical strains of M. abscessus. A narrow MIC distribution range was obtained $(0.47-3.75 \mu \mathrm{g} / \mathrm{mL})$ and only four 2-fold diluted concentrations were covered. 36.67\% (22/60) and 41.67\% $(25 / 60)$ of the clinical isolates had MIC at $0.93 \mu \mathrm{g} / \mathrm{mL}$ or $1.83 \mu \mathrm{g} / \mathrm{mL}$, respectively. A study using CCCP as an experimental control previously occasionally detected the MIC value of CCCP against M. abscessus ATCC19977 at $0.4 \mu \mathrm{g} / \mathrm{mL},{ }^{18}$ which was in accordance with our results. In this study, both the in vitro and intracellular testing demonstrated that CCCP has good and concentrationdependent antimicrobial activity against M. abscessus. 
A

MOI 1:1

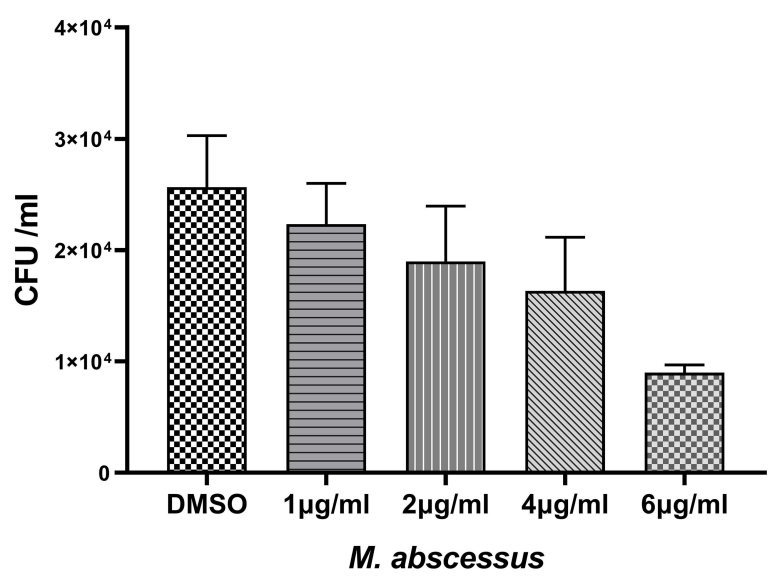

C

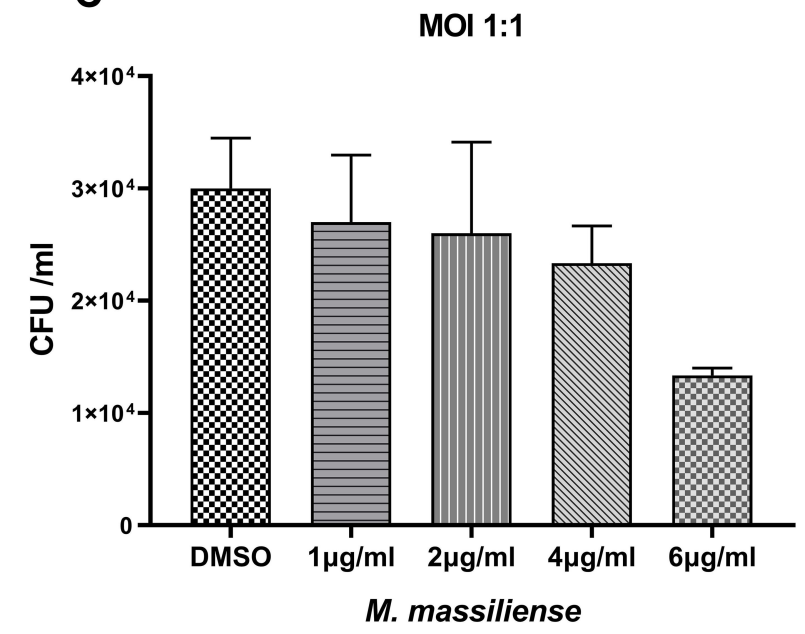

B

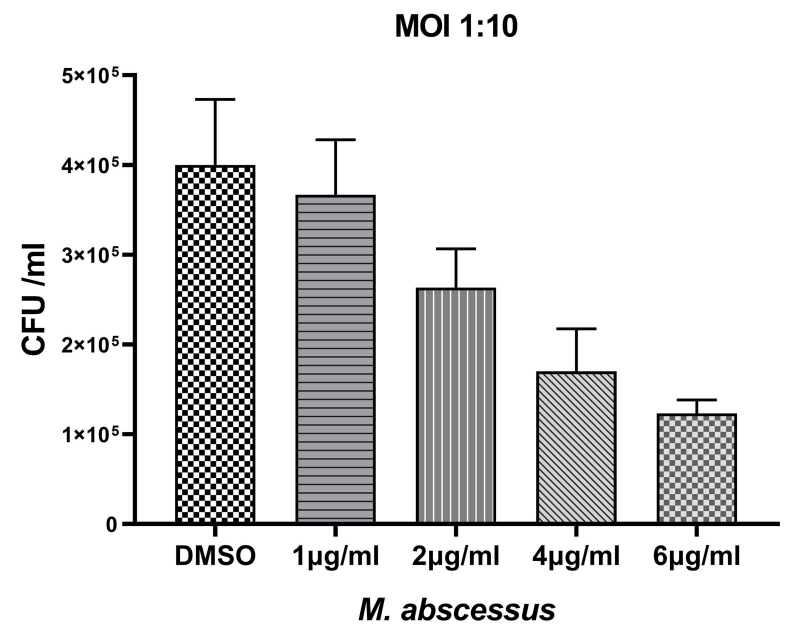

D

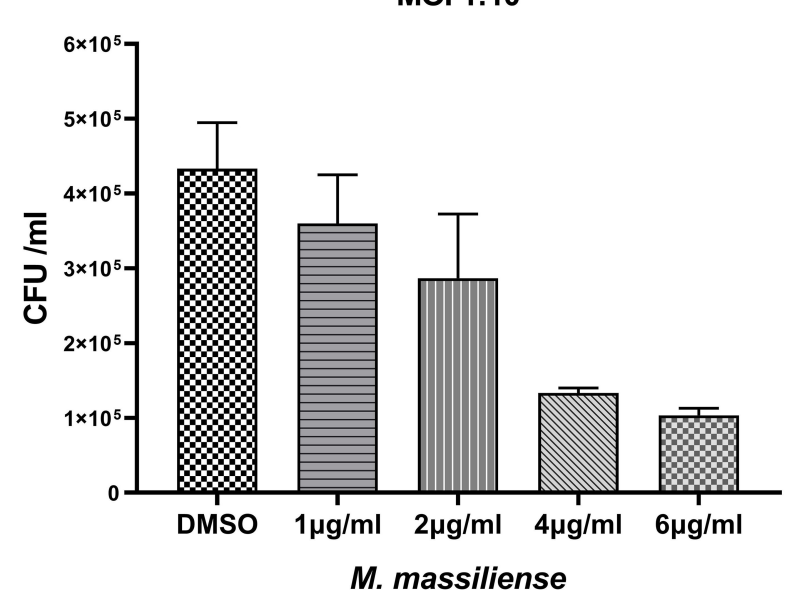

Figure 4 Intracellular bactericidal activities of different CCCP concentrations against $M$. abscessus and M. massilliense in macrophages. The number of $M$. abscessus (ATCC 19977) in macrophages treated with different concentrations of CCCP at a MOI of I (A) and a MOI of I0 (B); The number of M. massilliense (CCUG 48898) in macrophages treated with different concentrations of CCCP at a MOI of I (C) and MOI of 10 (D). All data are shown as the means \pm SD $(n=3)$.

Cytotoxicity assays indicated that THP-1 cells and U937 cells had high viability $(>97 \%)$ when exposed to CCCP $\leq 6.25 \mu \mathrm{g} / \mathrm{mL}$. The highest safe concentration was much greater than the greatest MIC of the enrolled M. abscessus clinical strains to CCCP, which favors the possibility of CCCP as a new antimicrobial agent. In the intracellular bactericidal experiments, CCCP plus CLA presented reasonable inhibitory activity in contrast to each of the drugs at doubled concentration. We presumed that the inhibition was mainly an outcome of the cumulative effect, while the possibility of the synergistic effect due to the canonical EPI function of CCCP was hard to be determined by our assay. We speculated that since the reference strains of M. abscessus were relatively susceptible to clarithromycin, which makes the efflux pump inhibition effect less obvious when it combined with CCCP.

M. abscessus, which consists of the three subspecies ie, M. abscessus subsp. abscessus, M. abscessus subsp. massiliense and $M$. abscessus subsp. bolletii, displays two colony morphology types: smooth and rough morphotypes. Previous studies reported that these three M. abscessus subspecies have different antibiotic resistance profiles. ${ }^{19}$ For example, $M$. abscessus subsp. massiliense was sensitive to doxycycline, but $M$. abscessus subsp. abscessus and M. abscessus subsp. bolletii were resistant to it. In addition, the sensitivities of the three subspecies to clarithromycin were not identical either. The major difference between M. abscessus subsp. abscessus and M. abscessus subsp. 
massiliense is that the latter does not have an intact erm(41) gene and thus does not have inducible macrolide resistance. Therefore, the treatment response may be better among patients infected with M. abscessus subsp. massiliense. ${ }^{20}$ Our study found that M. abscessus subspecies responded discrepantly when exposed to CCCP, the MIC for M. abscessus subsp. massiliense was generally one fold greater than this of M. abscessus subsp. abscessus. Since we do not have isolates of $M$. abscessus subsp. bolletii, we could not evaluate the activity of CCCP against this species.

Both smooth morphotype and rough morphotype can be found in subjects with $M$. abscessus infections. ${ }^{21}$ The smooth morphotype expresses glycopeptidolipid (GPL) on its cell surface while the rough morphotype expresses minimal amount of GPL on the cell wall. ${ }^{22-24}$ Biofilm formation and different colony morphotypes contribute to the persistence of M. abscessus in lung diseases. Correlations between morphotypes of M. abscessus and severity of disease were demonstrated in experimental and clinical studies, while rough morphotype has been considered more aggressive. ${ }^{25,26}$ However, the correlation between phenotype and drug susceptibility remains obscure. Gillian et al found that rough morphotype was more aggressive and formed biofilm-like aggregates, implying it was significantly more tolerant than smooth morphotype to acidic pHs and treatment with amikacin or azithromycin. ${ }^{27}$ Whereas several other studies did not find the colony morphology of $M$. abscessus having association with susceptibilities to clarithromycin, amikacin, or cefoxitin, and one study even found that the MIC of smooth morphotype was higher than rough morphotype to tigecycline. ${ }^{28}$ Our results showed that both morphotypes were susceptible to CCCP while smooth morphotype was more susceptible than rough morphotype. It would be an interesting study on whether CCCP has a stronger inhibitory activity against smooth morphotype resulting in a better therapeutic effect in vivo.

Our study has some limitations. Firstly, all the clinical isolates studied were obtained from a single institution. It may not represent all the characteristics of the $M$. abscessus isolates. Secondly, the bactericidal activity of CCCP against M. abscessus was only investigated in vitro and in macrophages, therefore the present result might not reflect the real reaction in vivo. Animal model study, pharmacokinetic/pharmacodynamic (PK/PD) studies and synergy of CCCP with other compounds should be further conducted to explore the antibacterial activity of CCCP against $M$. abscessus infection. Thirdly, although our result showed that the highest safe concentration was much greater than the greatest MIC value of the enrolled M. abscessus clinical strains, the toxicity of it is still a big concern. ${ }^{29-31}$ Due to its interfering with mitochondrial depolarization which would induce mitophagy process, applying CCCP in human should be extremely cautious.

In conclusion, $\mathrm{CCCP}$ has a strong antimicrobial activity against $M$. abscessus in vitro and in macrophages with less cytotoxicity. CCCP may be a promising drug candidate for $M$. abscessus treatment, for which very limited effective drug choice available nowadays.

\section{Funding}

The study was supported by National Major Science and Technology Projects of China (2018ZX10302-301-004), Beijing Natural Science Foundation (No.5192006), Tongzhou "Yun He" Talent Project (YHLD2018030), and Capital's Funds for Health Improvement and Research (CFH2020-4-2163), Beijing Municipal Administration of Hospitals Clinical Medicine Development of Special Funding Support (ZYLX201824), Beijing Hospitals Authority Youth Programme (QML20201601), and Beijing Municipal Administration of Hospitals' Ascent Plan (DFL20181602).

\section{Disclosure}

The authors declare no conflicts of interest.

\section{References}

1. Johansen MD, Herrmann JL, Kremer L. Non-tuberculous mycobacteria and the rise of mycobacterium abscessus. Nat Rev Microbiol. 2020;18(7):392-407. doi:10.1038/s41579-020-0331-1

2. Cowman $\mathrm{S}$, van Ingen J, Griffith DE, Loebinger MR. Non-tuberculous mycobacterial pulmonary disease. Eur Respir J. 2019;54(1):1900250. doi:10.1183/13993003.00250-2019

3. Koh WJ, Chang B, Jeong BH, et al. Increasing recovery of nontuberculous mycobacteria from respiratory specimens over a 10-year period in a tertiary referral hospital in south korea. Tuberc Respir Dis (Seoul). 2013;75(5):199-204. doi:10.4046/trd.2013.75.5.199

4. Griffith DE. Mycobacterium abscessus and antibiotic resistance: same as it ever was. Clin Infect Dis. 2019;69(10):1687-1689. doi:10.1093/ $\mathrm{cid} / \mathrm{ciz} 071$

5. Chen J, Zhao L, Mao Y, et al. Clinical efficacy and adverse effects of antibiotics used to treat mycobacterium abscessus pulmonary disease. Front Microbiol. 2019;10:1977. doi:10.3389/fmicb.2019.01977

6. Kwak N, Dalcolmo MP, Daley CL, et al. Mycobacterium abscessus pulmonary disease: individual patient data meta-analysis. Eur Respir J. 2019;54(1):1801991. doi:10.1183/13993003.01991-2018

7. Nessar R, Cambau E, Reyrat JM, Murray A, Gicquel B. Mycobacterium abscessus: a new antibiotic nightmare. J Antimicrob Chemother. 2012;67(4):810-818. doi:10.1093/jac/dkr578

8. Ardebili A, Talebi M, Azimi L, Rastegar Lari A. Effect of efflux pump inhibitor carbonyl cyanide 3-chlorophenylhydrazone on the minimum inhibitory concentration of ciprofloxacin in acinetobacter baumannii clinical isolates. Jundishapur $J$ Microbiol. 2014;7(1):e8691. doi: $10.5812 /$ jjm. 8691

9. Park YK, Ko KS. Effect of carbonyl cyanide 3-chlorophenylhydrazone (CCCP) on killing acinetobacter baumannii by colistin. $J$ Microbiol. 2015;53(1):53-59. doi:10.1007/s12275-015-4498-5 
10. Wang Y, Venter H, Ma S. Efflux pump inhibitors: a novel approach to combat efflux-mediated drug resistance in bacteria. Curr Drug Targets. 2016;17(6):702-719. doi:10.2174/1389450116666151001103948

11. Rodrigues L, Parish T, Balganesh M, Ainsa JA. Antituberculosis drugs: reducing efflux=increasing activity. Drug Discov Today. 2017;22(3):592-599. doi:10.1016/j.drudis.2017.01.002

12. Guo Q, Chen J, Zhang S, et al. Efflux pumps contribute to intrinsic clarithromycin resistance in clinical, mycobacterium abscessus isolates. Infect Drug Resist. 2020;13:447-454. doi:10.2147/IDR.S239850

13. Rindi L. Efflux pump inhibitors against nontuberculous mycobacteria. Int J Mol Sci. 2020;21(12):4191. doi:10.3390/ijms21124191

14. CLSI. Susceptibility Testing of Mycobacteria, Nocardia, and Other Aerobic Actinomycetes; Approved Standard, 2nd. Clsi Document M24-A2. CLSI; 2011.

15. Zhang S, Zou Y, Guo Q, et al. Ar-12 exhibits direct and host-targeted antibacterial activity toward mycobacterium abscessus. Antimicrob Agents Chemother. 2020;64(8). doi:10.1128/AAC.00236-20.

16. Viljoen A, Raynaud C, Johansen MD, et al. Verapamil improves the activity of bedaquiline against mycobacterium abscessus in vitro and in macrophages. Antimicrob Agents Chemother. 2019;63(9). doi:10.1128/AAC.00705-19.

17. Kasperbauer SH, De Groote MA. The treatment of rapidly growing mycobacterial infections. Clin Chest Med. 2015;36(1):67-78. doi:10.1016/j.ccm.2014.10.004

18. Ramis IB, Vianna JS, Silva Junior L, et al. In silico and in vitro evaluation of tetrahydropyridine compounds as efflux inhibitors in mycobacterium abscessus. Tuberculosis (Edinb). 2019;118:101853. doi:10.1016/j.tube.2019.07.004

19. Koh WJ, Jeon K, Lee NY, et al. Clinical significance of differentiation of mycobacterium massiliense from mycobacterium abscessus. Am J Respir Crit Care Med. 2011;183(3):405-410. doi:10.1164/ rccm.201003-03950C

20. Mougari F, Guglielmetti L, Raskine L, et al. Infections caused by Mycobacterium abscessus: epidemiology, diagnostic tools and treatment. Expert Rev Anti Infect Ther. 2016;14(12):1139-1154. doi:10.1080/14787210.2016.1238304

21. Catherinot E, Roux AL, Macheras E, et al. Acute respiratory failure involving an $\mathrm{r}$ variant of mycobacterium abscessus. J Clin Microbiol. 2009;47(1):271-274. doi:10.1128/JCM.01478-08

22. Nessar R, Reyrat JM, Davidson LB, Byrd TF. Deletion of the mmpl4b gene in the mycobacterium abscessus glycopeptidolipid biosynthetic pathway results in loss of surface colonization capability, but enhanced ability to replicate in human macrophages and stimulate their innate immune response. Microbiology (Reading). 2011;157(4):1187-1195. doi:10.1099/mic.0.046557-0
23. Pawlik A, Garnier G, Orgeur M, et al. Identification and characterization of the genetic changes responsible for the characteristic smooth-to-rough morphotype alterations of clinically persistent mycobacterium abscessus. Mol Microbiol. 2013;90(3):612-629. doi:10.1111/mmi.12387

24. Rhoades ER, Archambault AS, Greendyke R, et al. Mycobacterium abscessus glycopeptidolipids mask underlying cell wall phosphatidylmyo-inositol mannosides blocking induction of human macrophage TNF- $\alpha$ by preventing interaction with TLR2. J Immunol. 2009;183 (3):1997-2007. doi:10.4049/jimmunol.0802181

25. Li B, Ye M, Zhao L, et al. Glycopeptidolipid genotype correlates with the severity of mycobacterium abscessus lung disease. J Infect Dis. 2020;221(Supplement_2):S257-S262. doi:10.1093/infdis/jiz475

26. Rottman M, Catherinot E, Hochedez P, et al. Importance of $t$ cells, gamma interferon, and tumor necrosis factor in immune control of the rapid grower mycobacterium abscessus in $\mathrm{c} 57 \mathrm{bl} / 6$ mice. Infect Immun. 2007;75(12):5898-5907. doi:10.1128/IAI.00014-07

27. Clary G, Sasindran SJ, Nesbitt N, et al. Mycobacterium abscessus smooth and rough morphotypes form antimicrobial-tolerant biofilm phenotypes but are killed by acetic acid. Antimicrob Agents Chemother. 2018;62(3). doi:10.1128/AAC.01782-17.

28. Ruger K, Hampel A, Billig S, et al. Characterization of rough and smooth morphotypes of mycobacterium abscessus isolates from clinical specimens. J Clin Microbiol. 2014;52(1):244-250. doi:10.1128/ JCM.01249-13

29. Ni W, Li Y, Guan J, et al. Effects of efflux pump inhibitors on colistin resistance in multidrug-resistant gram-negative bacteria. Antimicrob Agents Chemother. 2016;60(5):3215-3218. doi:10.1128/AAC.00248-16

30. Mlejnek P. Caspase-3 activity and carbonyl cyanide m-chlorophenylhydrazone-induced apoptosis in HL-60. Altern Lab Anim. 2001;29(3):243-249. doi:10.1177/026119290102900313

31. Soutar MPM, Kempthorne L, Annuario E, et al. FBS/BSA media concentration determines CCCP's ability to depolarize mitochondria and activate PINK1-PRKN mitophagy. Autophagy. 2019;15 (11):2002-2011. doi:10.1080/15548627.2019.1603549
Infection and Drug Resistance

\section{Publish your work in this journal}

Infection and Drug Resistance is an international, peer-reviewed openaccess journal that focuses on the optimal treatment of infection (bacterial, fungal and viral) and the development and institution of preventive strategies to minimize the development and spread of resistance. The journal is specifically concerned with the epidemiology of antibiotic resistance and the mechanisms of resistance development and diffusion in both hospitals and the community. The manuscript management system is completely online and includes a very quick and fair peerreview system, which is all easy to use. Visit http://www.dovepress.com/ testimonials.php to read real quotes from published authors. 